\title{
Numerical Simulation of Interaction Between Red Blood Cell with Surrounding Fluid in Poiseuille Flow
}

\author{
Reza Esmaily*, Nader Pourmahmoud and Iraj Mirzaee \\ Department of Mechanical Engineering, Urmia University Pardis, Urmia, Iran
}

Received 14 October 2016; Accepted (in revised version) 3 May 2017

\begin{abstract}
In this research, motion and deformation of a red blood cell (RBC) in a microchannel with stenosis is investigated by combined Lattice Boltzmann-Immersed Boundary method. The fluid flow occurs due to the pressure difference between the inlet and the outlet of the microchannel. The immersed boundary algorithm guaranteed that there is no relative velocity between the RBC and fluid. Therefore, mass transfer along the immersed border does not occur. It can be seen that the healthy RBC has more deformation and passes the stenosis easier while the sick one passes the stenosis with less deformation and returns to its initial state faster. Increasing the pressure gradient (i.e., increasing Reynolds number) would cause more deformation of the RBC. It is found that a healthy RBC moves faster than a sick one along the microchannel. Blood pressure increases due to the presence of stenosis and low deformable RBCs.It is the reason of many serious diseases including cardiovascular diseases. The results of this paper were compared to the previous valid results and good agreements were observed.
\end{abstract}

AMS subject classifications: 74F99, 76M25

Key words: Interaction, Poiseuille flow, lattice Boltzmann method, numerical simulation.

\section{Introduction}

Blood is a non-homogeneous fluid that mainly consists of blood cells, plasma, and nutrients. Blood circulation in microvessels delivers oxygen and nutrients to living tissues and removes metabolic wastes. Red blood cells (RBCs) have biconcave discoid shapes of $8 \mathrm{~m}$ diameter and form 40 to $45 \%$ of the blood volume [1]. When the diameter of RBCs are comparable with the vessel dimensions of capillaries, the co-interaction of RBCs and the interaction between RBCs and plasma significantly affects the overall properties of

*Corresponding author.

Emails: reza_esmaily82@yahoo.com (R. Esmaily), n.pormahmod@urmia.ac.ir (N. Pourmahmoud), i.mirzaee@urmia.ac.ir (I. Mirzaee) 
blood [2]. For instance, the deformed shape of blood cells or the changes in plasma viscosity is a symptom of various diseases, such as acute myocardial infarction, malaria, and sickle cell anemia [3-5]. The RBC membrane can undergo large deformations and the cell shape is squeezed to a diameter of $3 \mathrm{~m}$ while flowing through the vessel. Deformed RBCs may increase blood viscosity and flow resistance, thus resulting in myocardial infarction and apoplexy [6].The circulation phenomenon can be aggravated under the presence of a pathological condition such as stenosis inside the microvessel, such as stenosis $[7,8]$.

Recently, the lattice Boltzmann method (LBM) in combination with IBM has been used for simulating the motion and deformation of elastic bodies immersed in fluid flow including red blood cells (RBCs). The LBM is fast, accurate, relatively simple, compatible with the desired geometries and highly parallelizable. Zhang et al. $[9,10]$ studied the dynamic behavior of RBC in shear flow and channel flow and investigated several hemodynamic and rheological properties, using a combination of LBM and IBM.. Cheng et al. [11] have proposed a proper model to simulate the fast boundary movements and a high pressure gradient occurred in the fluid-solid interaction. In their research mitral valve jet flow considering the interaction of leaflets and fluid has been simulated. Navidbakhsh and Rezazadeh [12] carried out a numerical study on the behavior of malaria-infected RBC. Vahidkhah and Abdollahi [13] simulated the motion of a massless elastic object in a twodimensional viscous channel flow numerically using IBM-LBM. Dadvand et al. [14,15] investigated numerically the motion and deformation of a RBC in a viscous shear flow utilizing a combined LBM-IBM. Due to the advances in micro-machine technology, experiments have been done on RBCs in a micro-channel with constriction [16-18]. Eggleton and Popel [19] combined immersed boundary method (IBM) with finite element method to simulate three-dimensional deformation of a RBC in a shear flow. Pozrikidis [20] has used boundary integral method to study motion and deformation of RBCs in the shear flow and the flow in the channel. Zhao et al. [21] have studied the time variations of RBCs deformation and flow resistance in the stenosed microchannels having a diameter less than $10 \mu \mathrm{m}$, using boundary integral method. Sun and Munn $[22,23]$ have studied RBC deformation of two-dimensional RBC in a $20-40 \mu \mathrm{m}$ channel using lattice Boltzmann method (LBM). They modeled the RBCs as two-dimensional solid particles. Bagchi [8] simulated a suspension containing multiple cells in the range of vessel size $20-30 \mu \mathrm{m}$ and discharge hematocrit 10-60\%, using IBM. Wang et al. [24] have used IB$\mathrm{M}$ with a spring model for simulating the blood flow in vessels with $8-11 \mu \mathrm{m}$ diameter and discharge hematocrit $10-41 \%$. They have also studied hydrodynamic hemorheologic properties such as umbrella shaped cells, flat velocity profile and Fahraeus effect. Xiong [25] used LBM-IBM to examine changes in the wall shear stress induced by RBC passing through micro-channel with 5-11 $\mu \mathrm{m}$ diameter. Li et al. [26] have used LBM for two-dimensional simulating of rigid particle suspensions in a stenosed microchannel. Hyakutake [27] conducted a two-dimensional simulation of the stenosed microvascular flow with rigid RBCs assuming primary pulmonary hypertension due to the stenosis of lung arteriole using LBM.

In the present article, motion and deformation of both healthy and sick RBC is inves- 
tigated in a microchannel with stenosis using Lattice Boltzmann method and Immersed Boundary method.The results of this paper were compared to the available results and good agreements were observed.

\section{Governing equations}

It was mentioned in introduction that in the IBM the fluid is represented on an Eulerian coordinate and the structure is represented on a Lagrangian coordinate. A typical two-dimensional example of an elastic solid membrane with curved boundary has been shown in Fig. 1. Consider a flexible solid membrane with the curved boundary $\Gamma$ immersed in the two-dimensional incompressible viscous fluid domain $\Omega$. The membrane boundary $\Gamma$ is characterized by the Lagrangian parameter $s$, and the fluid domain $\Omega$ is represented by Eulerian coordinates $\vec{x}$. Hence any point on the membrane can be written as $\vec{X}(s, t)$, where $s$ is arc length, and $t$ is time.

Hence, the equations governing the combination of fluid and solid motions are as following:

$$
\begin{aligned}
& \nabla \cdot \vec{u}=0, \\
& \rho\left(\frac{\partial \vec{u}}{\partial t}+\vec{u} \cdot \nabla \vec{u}\right)=-\nabla p+\eta \nabla^{2} \vec{u}+\vec{f}(\vec{x}, t), \\
& \vec{f}(\vec{x}, t)=\int_{\Gamma} \vec{F}(s, t) \delta(\vec{x}-\vec{X}(s, t)) d s .
\end{aligned}
$$

To satisfy the no-slip boundary condition on the fluid-solid interface, the velocity of any point on the solid surface must be equal to that of the adjacent fluid particle, i.e.,

$$
\vec{U}(s, t)=\vec{u}(\vec{X}(s, t), t)=\frac{\partial \vec{X}(s, t)}{\partial t}=\int_{\Gamma} \vec{u}(\vec{x}, t) \delta(\vec{x}-\vec{X}(s, t)) d \vec{x} .
$$

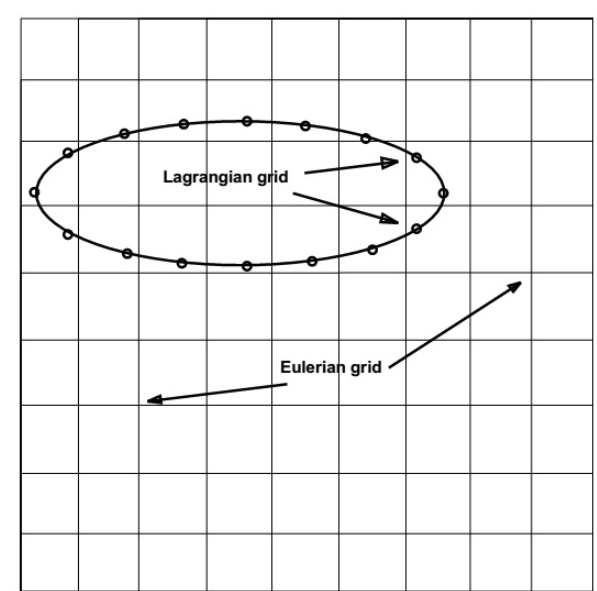

Figure 1: Schematic representation of immersed boundary (Lagrangian coordinates) and Eulerian mesh for fluid. 
In the above equations, $\rho$ and $\eta$ are the mass density and dynamic viscosity of the fluid, respectively. In addition, $\vec{u}$ and $p$ indicate the velocity and pressure fields, respectively. The term $\vec{f}$ on the right-side of Eq. (2.1b) denotes the RBC (Red Blood Cell) forces (tensile and bending) due to the elastic boundary immersed in the fluid.

Eq. (2.1c) indicates that the force density of the fluid $\vec{f}(\vec{x}, t)$ is obtained from the force density of the RBC $\vec{F}(s, t)$. Eq. (2.2) represents the no-slip condition at the fluid-solid interface, as the solid boundary moves with the same velocity as that of the surrounding fluid.

Mathematically the Dirac delta function $\delta(\vec{x})$ is discontinuous and has to be smoothed for numerical implementation:

$$
\delta(\vec{x})=\frac{1}{h^{2}} \Delta\left(\frac{x}{h}\right) \Delta\left(\frac{y}{h}\right),
$$

where

$$
\Delta(r) Z= \begin{cases}\frac{1}{8}\left(3-2 r+\sqrt{1+4 r-4 r^{2}}\right), & \text { for } 0 \leq r \leq 1 \\ \frac{1}{8}\left(5-2 r-\sqrt{-7+12 r-4 r^{2}}\right), & \text { for } 1<r \leq 2 \\ 0, & \text { for } 2<r\end{cases}
$$

where $h$ is the distance between two Eulerian grid points and $r$ denotes the distance between any two Eulerian and Lagrangian points.

In the IBM, to calculate the momentum exchange, the following collision function is used:

$$
f_{i}\left(\vec{x}+\hat{e}_{i} \Delta t, t+\Delta t\right)-f_{i}(\vec{x}, t)=-\frac{f_{i}(\vec{x}, t)-f_{i}^{e q}(\vec{x}, t)}{\tau}+\Delta t k_{i}
$$

where $f_{i}(\vec{x}, t)$ is the density distribution function of particles with the velocity $\hat{e}_{i}$ located at position $\vec{x}$ at time $t$. $\Delta t$ is time step, $f_{i}^{e q}(\vec{x}, t)$ is the equilibrium distribution function, $\tau$ indicates the dimensionless relaxation time and $k_{i}$ denotes the body force associated with the immersed body. In the present research, the LBM with two-dimensional model of D2Q9 has been used (see Fig. 2).

The particle velocity in the corresponding nine directions can be written as follows:

$$
\hat{e}_{i}= \begin{cases}(i, i), & i=0, \\ \left(\cos \frac{\pi}{2}(i-1), \sin \frac{\pi}{2}(i-1)\right) c, & i=1-4, \\ \sqrt{2}\left(\cos \frac{\pi}{2}(i-2 / 9), \sin \frac{\pi}{2}(i-2 / 9)\right) c, & i=5-8,\end{cases}
$$

where $c=\Delta x / \Delta t$. Here, $\Delta x$ is the distance between two successive nodes in the Euler grid. In the present research it is assumed that $\Delta x / \Delta t=1$. The equilibrium density distribution 


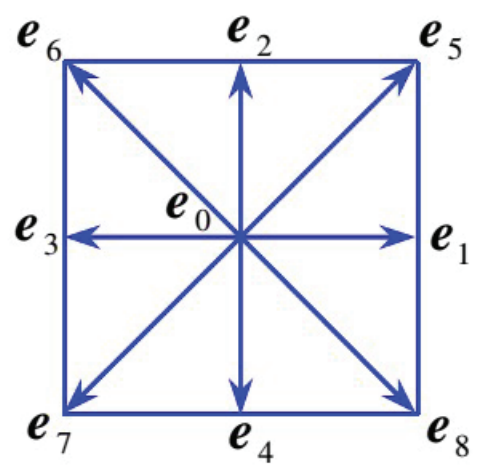

Figure 2: D2Q9 lattice model.

function is written as follow:

$$
f_{i}^{e q}(\vec{x}, t)=w_{i} \rho\left[1+3 \frac{\left(\hat{e}_{i} \cdot \vec{u}\right)}{C^{2}}+\frac{9}{2} \frac{\left(\hat{e}_{i} \cdot \vec{u}\right)^{2}}{C^{4}}-\frac{3}{2} \frac{|\vec{u}|^{2}}{C^{2}}\right] .
$$

The fluid pressure $p$ is calculated via an isothermal equation of state $\left(p=\rho C_{s}^{2}\right)$, where $\left(C_{s}=c / \sqrt{3}\right)$ is the speed of sound and $\rho$ is density. In addition, $w_{i}$ are weight coefficients with the following values,

$$
W_{i}= \begin{cases}4 / 9, & i=0, \\ 1 / 9, & i=1-4, \\ 1 / 36, & i=5-8 .\end{cases}
$$

The elastic force in the lattice Boltzmann equation $k_{i}$ is defined as,

$$
k_{i}=\left(1-\frac{1}{2 \tau}\right) W_{i}\left[3 \frac{\left(\hat{e}_{i} \cdot \vec{u}\right)}{C^{2}}+9 \frac{\left(\hat{e}_{i} \cdot \vec{u}\right)^{2}}{C^{4}} \hat{e}_{i}\right] \cdot \vec{f}_{i} .
$$

In addition, the density and microscopic fluid velocity are calculated from the following relations,

$$
\begin{aligned}
& \rho=\sum_{i=0}^{8} f_{i}, \\
& \vec{u}=\frac{1}{\rho}\left[\sum_{i=0}^{8} f_{i} \hat{e}_{i}+\frac{\Delta t}{2} \vec{f}\right] .
\end{aligned}
$$

The kinematic viscosity $v$ in the D2Q9 model is related to the dimensionless relaxation time $\tau$ as follows:

$$
v=C_{s}^{2}\left(\tau-\frac{1}{2}\right) \Delta t
$$


The Lagrangian force density $\vec{F}$ comprises two parts of tension-compression $\vec{F}_{s}$ and bending $\vec{F}_{b}$ forces, i.e.,

$$
\vec{F}(s, t)=\vec{F}_{s}(s, t)+\vec{F}_{b}(s, t)
$$

This force is related to the elastic potential energy density $\omega$ as follows (thanks to the virtual work theorem):

$$
\vec{F}(s, t)=-\frac{\partial \omega}{\partial \vec{X}}=-\frac{\partial\left(\omega_{s}+\omega_{b}\right)}{\partial \vec{X}}
$$

where

$$
w_{s}=\frac{1}{2} E_{s} \sum_{j=1}^{N-1}\left(\frac{\left|\vec{X}_{j+1}-\vec{X}_{j}\right|}{\Delta s}-1\right)^{2} \Delta s
$$

and

$$
w_{b}=\frac{1}{2} E_{b} \sum_{j=2}^{N-1}\left(\frac{\left|\vec{X}_{j+1}-2 \vec{X}_{j}+\vec{X}_{j-1}\right|}{(\Delta s)^{4}}\right)^{2} \Delta s .
$$

Here, $E_{s}$ and $E_{b}$ are elastic modulus (tension/compression constant) and bending modulus, respectively.

The discretized form of Lagrangian force density $\vec{F}$, and elastic potential energy density will become,

$$
\begin{aligned}
& \left(\vec{F}_{s}\right)_{k}=\frac{E_{s}}{(\Delta s)^{2}} \sum_{j=1}^{N-1}\left\{\left(\left|\vec{X}_{j+1}-\vec{X}_{j}\right|-\Delta s\right) \times \frac{\vec{X}_{j+1}-\vec{X}_{j}}{\left|\vec{X}_{j+1}-\vec{X}_{j}\right|}\left(\delta_{j, k}-\delta_{j+1, k}\right\},\right. \\
& \left(\vec{F}_{b}\right)_{k}=\frac{E_{b}}{(\Delta s)^{4}} \sum_{j=2}^{N-1}\left\{\left(\vec{X}_{j+1}-2 \vec{X}_{j}+\vec{X}_{j-1}\right)\left(2 \delta_{j, k}-\delta_{j+1, k}-\delta_{j-1, k}\right)\right\} .
\end{aligned}
$$

In Eqs. (2.13)-(2.15b), $k=1,2, \cdots, N,(N$ is the total number of Lagrangian nodes on the RBC), $\left(\vec{F}_{s}\right)_{k}$ and $\left(\vec{F}_{b}\right)_{k}$ are elastic Lagrangian forces associated with the node $k$ on the RBC and $\delta_{j, k}$ is the Kronecker delta function.

When the Lagrangian forces on the RBC are calculated, all the translational and rotational speeds are updated explicitly. It should be noted that the solid RBC moves continuously based on Newtonian dynamics and finally the new position of the membrane is obtained.

In the present work, the blood is considered as Newtonian fluids. The Newtonian nature of these fluids has been well estimated [2]. The non-Newtonian behaviour of blood is mainly due to the deformation of RBCs [8]. 
The time-averaged pressure $\bar{p}$, the Re number and other dimensionless parameters are defined as follows:

$$
\begin{array}{lll}
\bar{p}=\frac{1}{t_{2}-t_{1}} \int_{t_{1}}^{t_{2}} p(t) d t, & R e=\frac{\rho u_{\max } H}{\mu}, & x=\frac{x^{*}}{H}, \\
y=\frac{y^{*}}{H}, & p=\frac{\bar{p}}{p_{\text {out }}-p_{\text {in }}}, & t=\frac{t^{*} u_{\max }}{H} .
\end{array}
$$

Where $x, y, p$ and $t$ denote respectively the dimensionless vertical coordinate, horizontal coordinate, flow pressure and time. In addition, $H, u_{\max }, p_{\text {out }}$ and $p_{\text {in }}$ are the height of microchannel, the maximum velocity of the Poiseuille flow and the outlet and inlet pressures of the microchannel, respectively.

In the absence of external forces, a RBC assumes a bi-concave disk, the surface to volume ratio of which is considerably greater than that of a sphere and it can easily pass through the capillaries [28]. The cross-section profile of a RBC in $x-y$ plane is given by the following relation [29]:

$$
x=\sin \theta, \quad y=\cos \theta\left[0.207+2.002 \sin \theta-1.122 \sin ^{2} \theta\right], \quad 0<\theta<2 \pi .
$$

A modified periodic boundary condition is used at the inlet and outlet boundaries of the microvessel. To apply pressure gradient in Boltzmann method two methods can be used. In the first method pressure gradient will be replaced by an equivalent volumetric force. In the second method modified periodic boundary condition is used. But the use of equivalent volumetric force, has good agreement for uniform channels. Using modified periodic boundary condition has more precision in channels with uniform cross section [30].

The so-called bounce back boundary condition is imposed on the straight parts of microvessel wall and the Bouzidi-Firdaouss-Lallemand [31] boundary condition is applied on the curved parts of the microvessel walls.

\section{Validation}

For a membrane in the shear flow the tumbling motion has been studied by previous researches both experimentally and numerically [32-35]. In this section, to compare our results with the numerical results of Bagchi result [8], a microchannel with the length of $L=2500 \mu \mathrm{m}$, the height of $H=40 \mu \mathrm{m}$ involving a membrane with the diameter of $D=8 \mu \mathrm{m}$ and the elastics and bending moduli of $E_{s}=6 \times 10^{-6} \mathrm{~N} / \mathrm{m}, E_{b}=72 \times 10^{-19} \mathrm{~N} \cdot \mathrm{m}$, respectively is considered. The Reynolds number is considered equal to 0.07 and the flow viscosity is set to $0.001 \mathrm{~Pa} \cdot \mathrm{s}$. In Fig. 3(a) the tumbling motion of the membrane due to its high hardness has been illustrated. In Figs. 3(b), (c) and (d) the present numerical results have been compared with numerical results of Bagchi result [8], which represent good agreements. The vertical migration of the membrane's centroid has been depicted in Fig. 3(b). In general, the vertical migration of the membrane is a very slow process. The membrane 


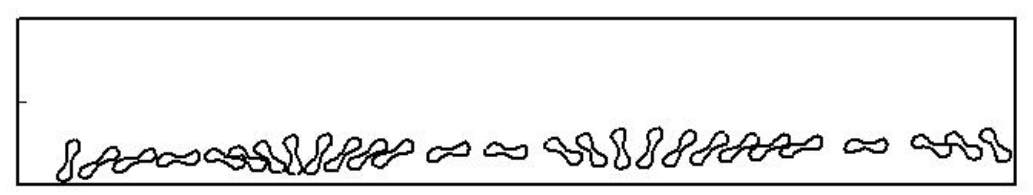

(a)

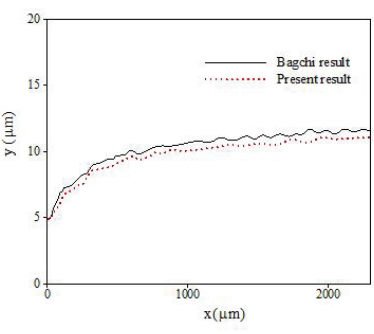

(b)

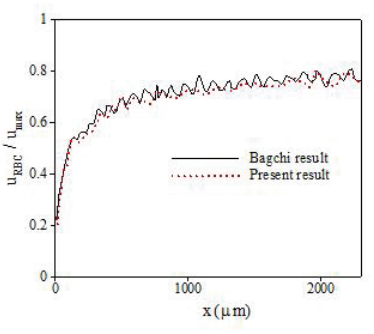

(c)

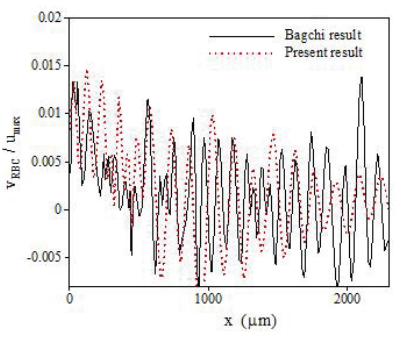

(d)

Figure 3: Tumbling motion of a membrane in Poiseuille flow (a), vertical displacement (b), horizontal velocity component $(c)$, vertical velocity component $(d)$ of the membrane.

experiences a displacement of $10 \mu \mathrm{m}$ along the vertical direction, while it displaces about $2500 \mu \mathrm{m}$ in the longitudinal direction. The migration degree depends on the deformability of membrane. The horizontal and vertical components of the membrane velocity are shown in Figs. 3(c) and (d), respectively. The velocity oscillations are due to the tumbling motion of the membrane. These oscillations elevate as the deformability of the membrane decreases. The negative values of the vertical component of the membrane velocity is because of its downward motion.

\section{Results and discussion}

Consider a Poiseuille flow between two walls. The fluid velocity at the walls is taken to be zero. Flow occurs due to pressure gradient between the inlet and the outlet of the microchannel. The constant values are given in Table 1. The Reynolds number is considered to be 0.35 .

Table 1: Grid dimensions and physical properties.

\begin{tabular}{||l|l||}
\hline Number of Eulerian points in the $x$-direction & 500 \\
Number of Eulerian points in the $y$-direction & 100 \\
Number of Lagrangian points & 60 \\
Time step & $1.2 \mu \mathrm{s}$ \\
relaxation time & 1 \\
Fluid density & $1000 \mathrm{~kg} / \mathrm{m}^{3}[10]$ \\
Plasma viscosity & $1.2 \mathrm{cp} \mathrm{[10]}$ \\
\hline
\end{tabular}




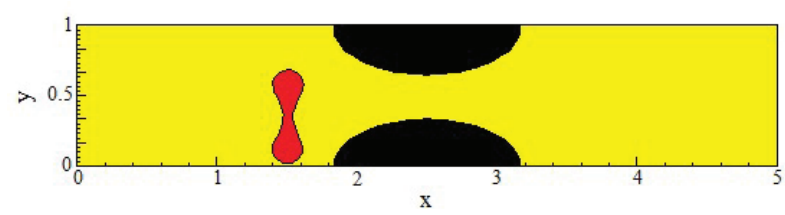

Figure 4: Geometry and nomenclature of the microchannel with stenosis.

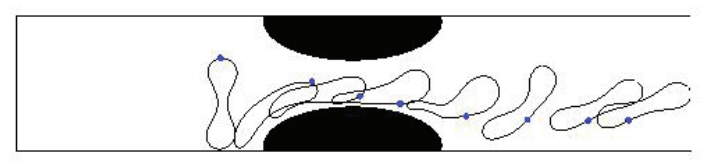

Figure 5: Motion and deformation of a healthy (high deformable) RBC through a microchannel with stenosis.

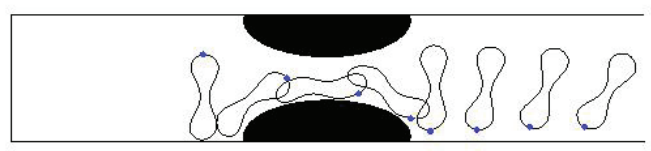

Figure 6: Motion and deformation of a sick (low deformable) RBC through a microchannel with stenosis.

Fig. 4 show the initial position of the RBC which is located below the central line of microchannel. The RBC length in vertical direction is considered 65 Eulerian lattice unit. Due to the force inserted to RBC from flow, Lagrangian grids of RBC shifts. It produces Lagrangian force. The Eulerian force inserted to the flow around the RBC is obtained with Eq. (2.1c). At the beginning of the solution RBC is suddenly released into the microchannels. In other words, RBC starts to move with a speed equal to developed flow at habitat and there is no relative speed between the cell and flow. Before he RBC released the flow has been developed. In fact, the flow has been assumed developed Poiseuille.

In Fig. 5 the healthy RBC is considered, which has the elastics moduli of $6 \times 10^{-6} \mathrm{~N} / \mathrm{m}$ and the bending moduli of $2 \times 10^{-19} \mathrm{~N} \cdot \mathrm{m}$. In Fig. 6, the sick RBC is considered, which has the elastics moduli of $6 \times 10^{-5} \mathrm{~N} / \mathrm{m}$ and the bending moduli of $4 \times 10^{-18} \mathrm{~N} \cdot \mathrm{m}$. These values are identical to those given in $[36,37]$.

The obtained results display an increment in the velocity of blood flow in the stenosis part. According to this fact, more deformations of the RBC would take place at this section. Both tank- treading and tumbling phenomena due to stiffness of the sick RBC can be seen in in Figs. 5 and 6, respectively [32]. The circular symbol on the RBC illustrates its rotation.

In the case, which the RBC is put below the center line of microchannel; it does not collide with the bottom walls of the stenosis (in order to avoid numerical instability, there 


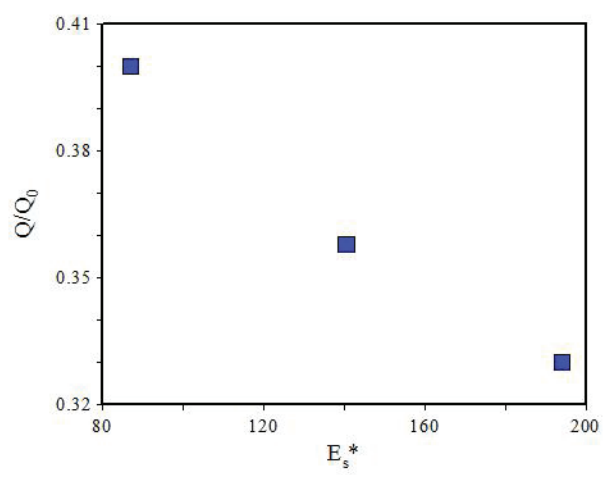

Figure 7: Variations of non-dimensional volumetric flow rate.

is at least one lattice unit distance between the microchannel and RBC's walls). Because of the obstacle existence in the way of the RBC in this case, the RBC inevitably moves towards the center of microchannel and stenosis. It can be seen that the healthy RBC has more deformation and passes the stenosis easier while the sick one is passed the stenosis with less deformation and returned to its initial position faster. This conclusion is in agreement with Hyakutake and Hongo [38] findings.

Healthy RBC has higher velocity than sick RBC [25,39]. Due to the existence of stenosis, more losses are appeared and consequently velocity of the RBC is reduced. Existence of stenosis decreases the velocity of the RBC before and after the stenosis and causes increment of its value during passing through the stenosis. Healthy and sick RBCs reach the end of michrochannel in time duration of 0.4 and 1.7 respectively. It should be noted that fast or slow motion of the RBC with respect to its normal state, causes inappropriate exchange of materials between the cell wall and the surrounding flow, which deteriorates its most important task of exchanging the materials with tissues.

Fig. 7 demonstrates the variations of non-dimensional volumetric flow rate with respect to the non-dimensional elastic modulus of $\operatorname{RBC}\left(E_{s}^{*}=E_{s} / \rho u_{\max }^{2} H\right)$ in a michrochannel with stenosis at $x=2.5$. The flow rate is defined as $Q=\int u d y$. The subscript " 0 " in Fig. 7 refers to the microchannel without RBC. In addition, the relation between flow rate and other parameters in pure plasma fluid (without RBC) is expressed as follow as $Q_{0}=\frac{\Delta p H^{3}}{12 \mu L}$, where $\Delta p, \mu, H$ and $L$ are pressure difference between the inlet and outlet, pure plasma viscosity, height and length of the microchannel, respectively [36]. Sick RBC produces a greater region of flow passage blocked causing a decrease in the flow velocity around the RBC. As a result, the volumetric flow rate decreases and the apparent viscosity and flow resistance increase. Increment of blood viscosity is indication of a disease named sickle cell anemia, where RBCs become sickle and stiffer and hence lose their ability of carrying oxygen [3].

Creation of stenosis in vessel causes the speed of blood transformation decrease and the process of supply of oxygen by RBCs become a serious problem for patient and blood 


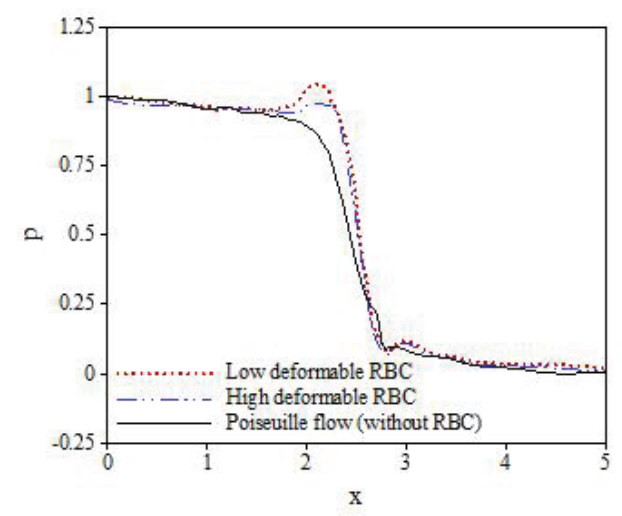

Figure 8: Time-averaged flow pressure at $y=0.5$.

pressure is increased. If this problem does not be resolved, it would cause physical problems for patient since the blood can hardly be transferred to other vessels. More cardiovascular diseases are relatedto the abnormal blood flow rate such as anemia ischemia caused by occurring vessel obstruction.It is observed that if the amount of stenosis increased, RBCs that have high stiffness andlow flexibility pass blocked vessels hardly. In addition, they block the other cells' path. If this process continues, it causes the risk of heart attack.

Fig. 8 displays the dimensionless time-averaged pressure of the flow in the microchannel with stenosis at $y=0.5$. Pressure also has been normalized with the pressure difference between the inlet and outlet of the microchannel. In the case of pure plasma motion in the microchannel (without RBC), the pressure decreases along the vessel due to frictional losses. For Sick RBC, the flow passage is more blocked due to the lack of flexibility of the $\mathrm{RBC}$ and hence it causes the flow velocity to decrease. As a result, the pressure around the RBC increases and becomes even higher than the inlet pressure of microchannel. This increased pressure is the reason of many serious diseases including cardiovascular diseases.

In Fig. 9, Lagrangian points velocity vector of the healthy $\mathrm{RBC}$ (at $t=0.8$ ) and the sick $\mathrm{RBC}$ (at $t=1.2$ ) at the exit region of the stenosis is observed. The RBC center is located at $x=3.3$, approximately. The Healthy RBC adapts itself easily with the flow and deforms more easily. The sick RBC have tumbling motion because of its more stiffness and it is almost vertical while exiting from stenosis. This way that sick cells exit from stenosis, slow down the flow velocity at stenosis and increase the blood pressure in this part of microchannel (Fig. 8). The reason of the slowdown is that the sick RBC blocks larger amount of patient blood flow.

In Fig. 10 horizontal component of flow velocity contour at time $t=0.25$ (Fig. 10(a)) and at time $t=0.8$ (Fig. 10(b)) is shown. One can see in Fig. 10(a) the healthy RBC adopt itself with flow because of higher deformability and passes more easily. Unlike as can be seen from Fig. 10(b), the flow velocity around the sick RBC is lower; meaning that 


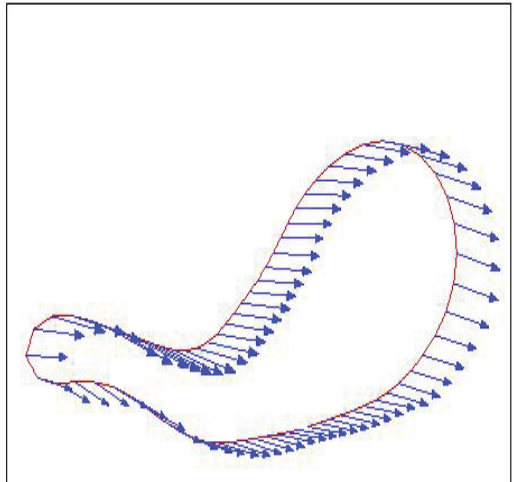

(a)

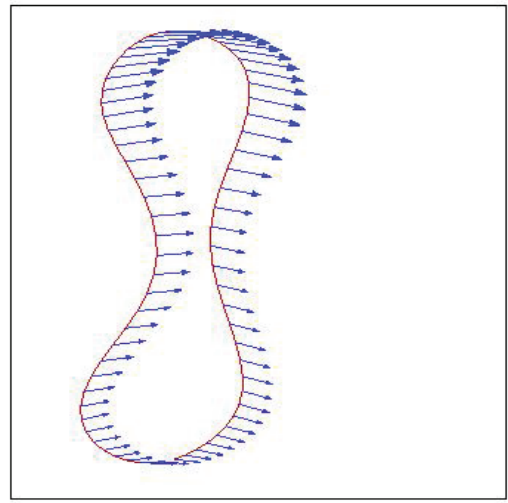

(b)

Figure 9: Velocity vector of lagrangian points (a) healthy RBC and (b) sick RBC at time passing the stenosis.

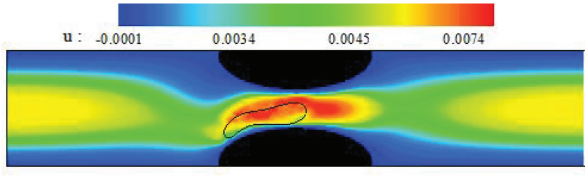

(a)

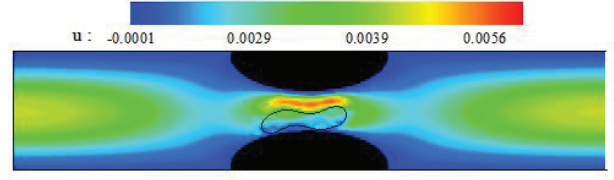

(b)

Figure 10: Horizontal component of flow velocity contour for (a) the healthy RBC and (b) the sick RBC.

the sick RBC is more rigid and barricades against the flow in stenosis part. This event in turn increases the blood pressure in the stenosis area as it can be seen in Fig. 8. Immersed boundary algorithm ensures that there is no relative speed between the RBC wall and the fluid. So immersed mass transfer does not occur along the boundary. RBC is assumed for a ring which the flow exists inside and outside it.

In Fig. 11, the effect of Reynolds number increment (i.e., $R e=3.5$ ) on deformation of the sick RBC (Fig. 6) is shown. The more the Reynolds the more deformation of RBC is achieved due to increase pressure difference between inlet and outlet of microchannel. So the RBC can pass the stenosis easier and faster. In fact, more shear force applied from fluid on the RBC which causes the RBC to become more stretch in the longitudinal direction.

\section{Conclusions}

In this manuscript, a hybrid LBM-IBM is used to simulate the hydrodynamic interaction of RBCs having different elastic moduli in a stenosed microchannel. The RBCs are con- 


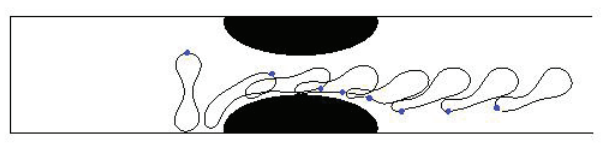

Figure 11: The effect increases of Reynolds number on deformation of the sick RBC.

sidered as elastic boundaries immersed in the fluid flow and represented in Lagrangian coordinates. The coupling method between the fluid and solid membranes is based on the IBM, which uses a uniform and fixed Eulerian mesh and removes the burden of expensive mesh updating in the traditional Arbitrary Lagrangian Eulerian (ALE) approach. The results were found to be in good agreement with available previous data. The present obtained results showed that healthy RBC moves faster than the sick one. Blood pressure increases at the presence of stenosis and low deformable $\mathrm{RBC}$, which is the reason of many serious diseases including cardiovascular diseases. It was seen that the healthy $\mathrm{RBC}$ had more deformation and passed the stenosis easier while the sick one passed the stenosis with less deformation and returned to initial state faster. Finally the effect of Reynolds number increasing was studied that illustrated the pressure gradient (increasing Reynolds number) would cause more deformation of the RBC.

\section{Acknowledgements}

This research did not receive any specific grant from funding agencies in the public, commercial, or not-for-profit sectors.

\section{References}

[1] J. F. Stolz, M. Singh And P.Riha, Hemorheology in Practice, IOS Press, Amsterdam, 1999.

[2] Y. C. FunG, Biomechanics: Mechanical Properties of Living Tissues, Springer-Verlag, New York,1993.

[3] A. S. Popel And P. C.Johnson, Microcirculation and hemorheology, Annu. Rev. Fluid. Mech., 37 (2005), pp. 43-69.

[4] H. S.Gokturk, M. Demir, N. A. Ozturk, G. K. Unler, S. Kulaksizoglu, I. KozANOGlu, E. Serin, AND U. YILMAZ, Plasmaviscosity changes in patients with liver cirrhosis, South Med. J., 102 (2009), pp. 1013-1018.

[5] H. A. Cranston, C. W. Boylan, G. L. Carroll, S. P. Sutera, J. R. Williamson, I. Y. GLUZMAN, AND D. J. KROGSTAD, Plasmodiumfalciparum maturation abolishes physiologic red cell deformability, Science, 223 (1984), pp. 400-403.

[6] X. Q. MI, J. Y. CHEN AND L. W. ZHOU, Effect of low power laser irradiation on disconnecting the membrane attached hemoglobin from erythrocyte membrane, J. Photochem. Photobiol. B Biol., 83 (2006), pp. 146-150. 
[7] C. POzRIKIDIs, Numerical simulation of the flow-induced deformation of red blood cells, Ann. Biomed. Eng., 31 (2003), pp. 1194-1205.

[8] P. BAGCHI, Mesoscale simulation of blood flow in small vessels, Biophys. J., 92 (2007), pp. 18581877.

[9] J. F. Zhang, P. C. Johnson And A. S. Popel, An immersed boundary lattice Boltzmann approach to simulate deformable liquid capsules and its application to microscopic blood flows, Phys. Biol., 4 (2007), pp. 285-295.

[10] J. F. ZhANG, P. C. JOHNSON AND A. S. POPEL, Red blood cell aggregation and dissociation in shear flows simulated by lattice Boltzmann method, J. Biomech, 41 (2008), pp. 47-55.

[11] Y. CHENG AND H. ZHANG, Immersed boundary method and lattice Boltzmann method coupled FSI simulation of mitral leaflet flow, Comput. Fluids, 39 (2010), pp. 871-881.

[12] M. NAVIDBAKHSH AND M. ReZAZADEH, An immersed boundary-lattice Boltzmann model for simulation of malaria-infected red blood cell in micro-channel, Sci. Iranica., 19 (2012), pp. 13291336.

[13] K. VAhidKhah AND V. ABDollahi, Numerical simulation of a flexible fiber deformation in a viscous flow by the immersed boundary-lattice Boltzmann method, Commun. Nonlinear Sci. Numer. Simulation, 17 (2012), pp. 1475-1484.

[14] A. AlizAdeH AND A. DADVAND, Siulatmion of the motion of two elastic membranes in Poiseuille shear flow via a combined immersed boundary-lattice Boltzmann method, J. Comput. Sci., 12 (2016), pp. 51-61.

[15] A. Dadvand, M. Baghalnezhad, I. Mirzaee, B. C. Khoo and S. Ghoreishi, An immersed boundarylattice Boltzmann approach to study the dynamics of elastic membranes in viscous shear flows, J. Comput. Sci., 5 (2014), pp. 709-718.

[16] M. Faivre, M. AbKarian, K. Bickraj And A. Stone, Geometrical focusing of cells in a microfluidic device: An approach to separate blood plasma, Biorheology, 43 (2006), pp. 147-159.

[17] M. KANG, H. S. JI AND K. C. KIM, Invivo investigation of RBC's flow characteristics and hemodynamics feature through a microchannel with a micro-stenosis, Int. J. Bio. Biomed. Eng., 2 (2008), pp. 1-8.

[18] H. Fujiwara, T. Ishikawa, T. Lima, R. Matsuki, Y. Imai, H. Kaji, M. Nishizawa AND T. YAMAGUCHI, Red blood cell motions in high-hematocrit blood flowing through a stenosed microchannel, J. Biomech., 42 (2009), pp. 838-843.

[19] C. D. EGGLETON AND A. S. POPEL, Large deformation of red blood cell ghosts in a simple shear ow, Phys. Fluids, 10 (1998), pp. 1834-1845.

[20] C. POZRIKIDIS, Finite deformation of liquid capsules enclosed by elastic membranes in simple shear ow, J. Fluid. Mech., 297 (1995), pp. 123-152.

[21] H. ZhaO, A Isfahani, L. Olson AND J. Freund, A spectral boundary integral method for flowing blood cells, J. Comput. Phys., 229 (2010), pp. 3726-3744.

[22] C. Sun, C. Migliorini AND L. MunN, Red blood cells initiate leukocyte rolling in post capillary expansions: a lattice Boltzmann analysis, Biophys. J., 85 (2003), pp. 208-222.

[23] C. Sun AND L. MunN, Particulate nature of blood determines macroscopic rheology: A 2-D lattice Boltzmann analysis, Biophys. J., 88 (2005), pp. 1635-1645.

[24] T. WANG AND Z. XING, Characterization of blood ow in capillaries by numerical simulation, J. Modern. Phys., 1 (2010), pp. 335-349.

[25] W. XIONG AND J. ZHANG, Shear stress variation induced by red blood cell motion in microchannel, Ann. Biomed. Eng., 38 (2010), pp. 2649-2659.

[26] H. LI, H. FANG, Z. LIN, S. XU AND S. CHEN, Lattice Boltzmann simulation on particle suspensions in a two-dimensional symmetric stenotic artery, Phys. Rev. E., 69 (2004), pp. 031919-031928. 
[27] T. HYAKUTAKE, S.OHKAWA, S. MOHRI AND S. YANASE, Lattice Boltzmann analysis of microvascular constriction flow including red blood cell and liposome-encapsulated hemoglobin, Theor. App. Mech. Japan, 56 (2008), pp. 215-224.

[28] X. HE AND L. LUO, Theory of the lattice Boltzmann method: from the Boltzmann equation to the lattice Boltzmann equation, Phys. Rev. E, 56 (1997), pp. 6811-6817.

[29] Y. SuI, X. CHEN, Y. CHEW, P. ROY AND H. LOW, Numerical simulation of capsule deformation in simple shear flow, Comput. Fluids, 39 (2010), pp. 242-250.

[30] J. ZHANG AND D. KWOK, Pressure boundary condition of the lattice Boltzmannmethod for fully developed periodic flows, Phys. Rev. E., 73 (2006), pp. 047702-047706.

[31] M. BOUZIDI, M. FIRDAOUSS AND P. LALLEMAND, Momentum transfer of a Boltzmann-lattice fluid with boundaries, Phys. Fluids, 13 (2001), pp. 3452-3459.

[32] T. FisCHER, M. STOHR-LisSEN AND H. SCHMID-SCHONBEIN, The red cell as a fluid droplet: tank tread-like motion of the human erythrocyte membrane in shear flow, Science, 202 (1978), pp. 894-896.

[33] H. Goldsmith, Red cell motions and wall interactions in tube ow, Federal. Proc., 30 (1971), pp. 1578-1590.

[34] C. POZRIKIDIS, Numerical simulation of the ow-induced deformation of red blood cells, Ann. Biomed. Eng., 37 (2003), pp. 1194-1205.

[35] S. R. KELLER AND R. SKALAK, Motion of a tank-treading ellipsoidal particle in a shear flow, J. Fluid Mech., 120 (1982), pp. 27-47.

[36] J. F. ZHANG, P. C. JOHNSON AND A. S. POPEL, Effects of erythrocyte deformability and aggregation on the cell free layer and apparent viscosity of microscopic blood ows, Microvascular Res., 77 (2009), pp. 265-272.

[37] D. A. FEDOSOV, Quantifying the biophysical characteristics of Plasmodium-falciparum-parasitized red blood cells in microcirculation, Proce. Nation. Aca. Sci., 108 (2011), pp. 35-39.

[38] T. HYAKUTAKE AND T. HONGO, Two-dimensional simulation of the flow behavior of a single deformable red blood cell suspension through a stenosed microvessel, J. Biomech. Sci. Eng., 9 (2014).

[39] G. MA, H. JINSONG AND L. HUA, Numerical modeling of the behavior of an elastic capsule in a microchannel flow: The initial motion, Phys. Rev. E, 79 (2009), pp. 046710-046717. 